Jurnal Natur Indonesia 13(2), Februari 2011: 94-99

ISSN 1410-9379, Keputusan Akreditasi No 65a/DIKTI/Kep./2008

\title{
Matriks atas Aljabar Max-Plus Interval
}

\author{
Marcellinus Andy Rudhito' ${ }^{*}$, Sri Wahyuni'2), Ari Suparwanto²), dan Frans Susilo ${ }^{3)}$ \\ 1) Jurusan Fakultas Matematika dan IImu Pengetahuan Alam, Fakultas Keguruan dan IImu Pendidikan, \\ Universitas Sanata Dharma, Jalan Paingan Maguwoharjo, Sleman, Yogyakarta 55283 \\ 2)Jurusan Matematika, Fakultas Matematika dan IImu Pengetahuan Alam, Universitas Gadjah Mada, \\ Jalan Sekip Utara Bulak Sumur 21, Yogyakarta 55281 \\ 3)Jurusan Matematika, Fakultas Sains dan Teknologi, Universitas Sanata Dharma, \\ Jalan Paingan Maguwoharjo, Sleman, Yogyakarta 55283
}

Diterima 23-04-2010

Disetujui 23-11-2010

\begin{abstract}
This paper aims to discuss the matrix algebra over interval max-plus algebra (interval matrix) and a method to simplify the computation of the operation of them. This matrix algebra is an extension of matrix algebra over maxplus algebra and can be used to discuss the matrix algebra over fuzzy number max-plus algebra via its alpha-cut. The finding shows that the set of all interval matrices together with the max-plus scalar multiplication operation and max-plus addition is a semimodule. The set of all square matrices over max-plus algebra together with an interval of max-plus addition operation and max-plus multiplication operation is a semiring idempotent. As reasoning for the interval matrix operations can be performed through the corresponding matrix interval, because that semimodule set of all interval matrices is isomorphic with semimodule the set of corresponding interval matrix, and the semiring set of all square interval matrices is isomorphic with semiring the set of the corresponding square interval matrix.
\end{abstract}

Keywords: idempotent, interval, matrix algebra, max-plus algebra, semiring

\section{PENDAHULUAN}

Pemodelan dan analisa suatu jaringan dengan pendekatan aljabar max-plus dapat memberikan hasil analitis dan lebih mudah pada komputasinya, Dalam Bacelli et al., (2001), Rudhito, (2004); dan Krivulin, (2001). Dalam masalah pemodelan dan analisa suatu jaringan di mana waktu aktivitasnya belum diketahui, misalkan karena masih pada tahap perancangan, datadata mengenai waktu aktivitas belum diketahui secara pasti maupun distribusinya. Waktu aktivitas ini dapat diperkirakan berdasarkan pengalaman maupun pendapat dari para ahli maupun operator jaringan tersebut. Untuk itu waktu aktifitas jaringan dimodelkan dalam suatu bilangan kabur (fuzzy number). Akhir-akhir ini telah berkembang pemodelan jaringan yang melibatkan bilangan kabur. Untuk masalah penjadwalan yang melibatkan bilangan kabur dapat dilihat pada Chanas dan Zielinski, (2001), sedangkan untuk masalah model jaringan antrian yang melibatkan bilangan kabur dapat dilihat pada Luthi dan Haring, (1997).

\footnotetext{
*Telp: +6285842842425

Email: rudhito@staff.usd.ac.id
}

Pemodelan dan analisa suatu sistem jaringan yang melibatkan bilangan kabur, sejauh penulis ketahui, belum ada yang menggunakan pendekatan aljabar maxplus. Operasi-operasi pada bilangan kabur dapat dilakukan menggunakan potongan-potongan- $\alpha-n y a$ yang didasarkan pada Teorema Dekomposisi dalam himpunan kabur (Susilo, 2006). Rudhito et al., (2008), telah dibahas suatu aljabar dengan elemen-elemennya berupa interval dengan operasi maximum dan penjumlahan yang didefinisikan di dalamnya. Pemodelan jaringan dengan pendekatan aljabar maxplus, graf untuk jaringan dinyatakan dengan menggunakan matriks, dengan unsur-unsurnya menyatakan waktu aktifitas antar titik pada jaringan tersebut. Dengan demikian pemodelan jaringan dengan waktu aktifitasnya yang berupa bilangan kabur, dengan pendekatan aljabar max-plus, akan terkait dengan matriks yang unsur-unsurnya berupa bilangan kabur. Untuk itu dalam makalah ini akan dibahas matriks atas aljabar max-plus interval, di mana operasi-operasinya merupakan perluasan dari operasi-operasi pada aljabar max-plus interval. Pada makalah ini akan dapat mempermudah pengoperasiannya. 
Aljabar Max-Plus dan Matriks Atas Aljabar Max-Plus. Dalam bagian ini dibahas konsep dasar matriks atas aljabar max-plus. Pembahasan selengkapnya dapat dilihat pada Baccelli et al., (1992) dan Rudhito, (2003).

$\mathbf{R}_{\varepsilon}:=\mathbf{R} \cup\{\varepsilon\}$ dengan $\mathbf{R}$ adalah himpunan semua bilangan real dan : $=-\infty$. Pada $\mathbf{R}_{\varepsilon}$ didefinisikan operasi berikut (Baccelli et al., (1992):

$\forall a, b \in \mathbf{R}_{\varepsilon}, a \oplus b:=\max (a, b)$ dan $a \otimes b:=a+b$.

Dapat ditunjukkan bahwa $\left(\mathbf{R}_{\varepsilon}, \oplus, \otimes\right)$ merupakan semiring idempoten komutatif dengan elemen netral $\varepsilon$ $=-\infty$ dan elemen satuan $e=0$. Lebih lanjut $\left(\mathbf{R}_{\varepsilon}, \oplus, \otimes\right)$ merupakan semifield yaitu bahwa $(\mathbf{R}, \oplus, \otimes)$ merupakan semiring komutatif di mana untuk setiap $a \in \mathbf{R}$, terdapat -a sehingga berlaku $a \otimes(-a)=0$. Kemudian $\left(\mathbf{R}_{\varepsilon}, \oplus, \otimes\right)$ disebut dengan aljabar max-plus, yang selanjutnya cukup dituliskan dengan $\mathbf{R}_{\max }$.

Dalam hal urutan pengoperasian (jika tanda kurang tidak dituliskan), operasi $\otimes$ mempunyai prioritas yang lebih tinggi dari pada operasi $\oplus$. Karena $\left(\mathbf{R}_{\max }, \oplus\right)$ merupakan semigrup komutatif idempoten, maka relasi " $\preceq_{m}$ " yang didefinisikan pada $\mathbf{R}_{\max }$ dengan $x \preceq_{m} y \Leftrightarrow x \oplus$ $y=y$ merupakan urutan parsial pada $\mathbf{R}_{\text {max }}$. Lebih lanjut relasi ini merupakan urutan total pada $\mathbf{R}_{\max }$. Karena $\mathbf{R}_{\text {max }}$ merupakan semiring idempoten, maka operasi $\oplus$ dan

$\otimes$ konsisten terhadap urutan $\preceq_{\mathrm{m}}$, yaitu $\forall a, b, c \in$ $\mathbf{R}_{\text {max }}$, jika $a \preceq_{\mathrm{m}} b$, maka $a \oplus c \preceq_{\mathrm{m}} b \oplus c$, dan $a \otimes c \preceq_{\mathrm{m}}$ $b \otimes c$. Aljabar max-pus $\mathbf{R}_{\max }$ tidak memuat pembagi nolyaitu $\forall x, y \in \mathbf{R}_{\varepsilon}$ berlaku: jika $x \otimes y=\varepsilon$ maka $x=$ $\varepsilon$ atau $y=\varepsilon$.

Operasi $\oplus$ dan $\otimes$ pada $\mathbf{R}_{\text {max }}$ di atas dapat diperluas untuk operasi-operasi matriks dalam $\mathbf{R}_{\max }^{m \times n}$ : $=\left\{A=\left(A_{i j}\right) \mid A_{i j} \in \mathbf{R}_{\text {max }}\right.$, untuk $i=1,2, \ldots, m$ dan $j=$ $1,2, \ldots, n\}$. Khususnya untuk $A, B \in \mathbf{R}_{\max }^{n \times n}$ dan $\alpha \in$ $\mathbf{R}_{\text {max }}$ didefinisikan ${ }_{n}(\alpha \otimes A)_{i j}=\alpha \otimes A_{i j},(A \oplus B)_{i j}=A_{i j} \oplus B_{i j}$ dan $(A \otimes B)_{i j}=\bigoplus_{k=1} A_{i k} \otimes B_{k j}$.

Dapat ditunjukkan bahwa $\left(\mathbf{R}_{\max }^{m \times n}, \oplus\right)$ merupakan semigrup komutatif idempotent dan $\left(\mathbf{R}_{\max }^{n \times n}, \oplus, \otimes\right)$ merupakan semiring idempoten. Relasi $\preceq_{m}$ yang didefinisikan pada $\mathbf{R}_{\text {max }}^{m \times n}$ dengan $A \preceq_{\mathrm{m}} B \Leftrightarrow A \oplus B=$ $B$ merupakan urutan parsial. Operasi $\oplus$ dan $\otimes$ konsisten terhadap urutan $\preceq_{m}$ dalam $\mathbf{R}_{\max }^{n \times n}$. Operasi $\oplus$ dan perkalian skalar $\otimes$ konsisten terhadap urutan dalam semimodul atas $\mathbf{R}_{\max }$.

Aljabar Max-Plus Interval. Dalam bagian ini dibahas konsep dasar aljabar max-plus interval. Pembahasan selengkapnya dapat dilihat pada Rudhito et al., (2008). Interval (tertutup) dalam $R_{\max }$ adalah suatu himpunan bagian dari $R_{\max }$ yang berbentuk $\mathbf{x}=[\underline{\mathrm{x}}, \overline{\mathrm{x}}]$

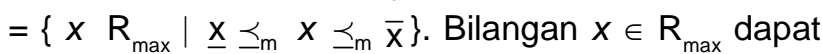
dinyatakan sebagai interval $x=[x, x]$. Didefinisikan $\mathrm{I}(\mathrm{R})_{\varepsilon}$ $=\left\{\mathrm{x}=[\underline{\mathrm{x}}, \overline{\mathrm{x}}] \mid \underline{\mathrm{x}}, \overline{\mathrm{x}} \in \mathrm{R}, \varepsilon \prec_{\mathrm{m}} \underline{\mathrm{x}} \underline{\mathrm{g}}_{\mathrm{m}} \overline{\mathrm{x}}\right\} \cup\{[\varepsilon, \varepsilon]\}$. Pada $\mathrm{I}(\mathrm{R})_{\varepsilon}$ didefinisikan operasi $\bar{\oplus}$ dan $\bar{\otimes}$ dengan (Litvinov \& Sobolevskii, 2001):

$x \bar{\oplus} y=[\underline{x} \oplus \underline{y}, \bar{x} \oplus \bar{y}]$ dan $x \bar{\otimes} y=[\underline{x} \otimes \underline{y}, \bar{x} \otimes \bar{y}]$ , $x, y \in I(R)_{\varepsilon}$.

Misalnya: $[-1,1] \bar{\oplus}[1,3]=[1,3]$ dan $[-1,1] \bar{\otimes}$ $[1,3]=[0,4]$.

Dapat ditunjukkan bahwa $\left(\mathbf{I}(\mathbf{R})_{\varepsilon}, \bar{\oplus}, \bar{\otimes}\right)$ merupakan semiring idempoten dengan elemen netral $\varepsilon=[\varepsilon, \varepsilon]$ dan elemen satuan $0=[0,0]$. Lebih lanjut $\left(\mathbf{I}(\mathbf{R})_{\varepsilon}, \bar{\oplus}, \bar{\otimes}\right)$ merupakan semiring idempoten komutatif. Selanjutnya $\left(\mathbf{I}(\mathbf{R})_{\varepsilon}, \bar{\oplus}, \bar{\otimes}\right)$ disebut dengan aljabar max-plus interval yang cukup dituliskan dengan $\mathbf{I}(\mathbf{R})_{\max }$.

Matriks atas Aljabar Max-Plus Interval I(R) max. $_{\text {. }}$ Bagian ini merupakan bagian utama pembahasan makalah ini. Operasi $\bar{\oplus}$ dan $\bar{\otimes}$ pada I $(R)_{\max }$ di atas dapat diperluas untuk operasi-operasi matriks dalam $\mathrm{I}(\mathrm{R})_{\max }^{m \times n}$ seperti dalam definisi berikut.

\section{Definisi 1.}

Didefinisikan $\mathrm{I}(\mathrm{R})_{\max }^{m \times n}:=\left\{\mathrm{A}=\left(\mathrm{A}_{i j}\right) \mid \mathrm{A}_{i j} \in \mathrm{I}(\mathrm{R})_{\text {max }}\right.$, untuk $i=1,2, \ldots, m, j=1,2, \ldots, n\}$. Matriks anggota $\mathrm{I}(\mathrm{R})_{\max }^{m \times n}$ disebut matriks interval max-plus.

\section{Definisi 2.}

Matriks $A, B \in I(R)_{\max }^{m \times n}$ dikatakan sama jika $A_{i j}=$ $\mathrm{B}_{i j}$, yaitu jika $\underline{\mathrm{A}_{i j}}=\underline{\mathrm{B}_{i j}}$ dan $\overline{\mathrm{A}_{i j}}=\overline{\mathrm{B}_{i j}}$ untuk setiap $i$ dan $j$.

\section{Definisi 3.}

i) Diketahui $\alpha \in \mathrm{I}(\mathrm{R})_{\max }, \mathrm{A}, \mathrm{B} \in \mathrm{I}(\mathrm{R})_{\max }^{\operatorname{m\times n}}$. Didefinisikan operasi perkalian skalar $\bar{\otimes}$ dengan $\alpha \bar{\otimes}$ $A$ adalah matriks yang unsur ke-ij-nya: $(\alpha \bar{\otimes} A)_{i j}=$ $\alpha \bar{\otimes} A_{i j}$, dan operasi $\bar{\oplus}$ dengan $A \bar{\oplus} B$ adalah matriks yang unsur ke-ij-nya : $(\mathrm{A} \Phi \mathrm{B})_{i j}=\mathrm{A}_{i j} \bar{\oplus} \mathrm{B}_{i j}$ untuk $i=1$, $2, \ldots, m$ dan $j=1,2, \ldots, n$.

ii) Diketahui $\mathrm{A} \in \mathbf{I}(\mathbf{R})_{\max }^{m \times p}, \quad B \in \mathbf{I}(\mathbf{R})_{\max }^{p \times n}$. Didefinisikan operasi $\bar{\otimes}$ dengan $A \bar{\otimes} B$ adalah matriks yang unsur ke-ij-nya: $(\mathrm{A} \bar{\otimes} \mathrm{B})_{i j}=\bigoplus_{k=1} \mathrm{~A}_{i k} \bar{\otimes} \mathrm{B}_{k j}$ untuk $i$ $=1,2, \ldots, m$ dan $j=1,2, \ldots, n$.

\section{Contoh 1}

Berikut diberikan beberapa contoh perhitungan operasi-operasi dalam matriks interval.

i). $[3,4] \quad \bar{\otimes} \quad\left[\begin{array}{cc}{[0,0]} & {[4,6]} \\ {[-1,1]} & {[\varepsilon, \varepsilon]} \\ {[0,1]} & {[-3,-2]}\end{array}\right]=$


$\left[\begin{array}{cc}{[3,4] \bar{\otimes}[0,0]} & {[3,4] \bar{\otimes}[4,6]} \\ {[3,4] \otimes[-1,1]} & {[3,4] \bar{\otimes}[\varepsilon, \varepsilon]} \\ {[3,4] \bar{\otimes}[0,1]} & {[3,4] \bar{\otimes}[-3,-2]}\end{array}\right]=\left[\begin{array}{cc}{[3,4]} & {[7,10]} \\ {[2,5]} & {[\varepsilon, \varepsilon]} \\ {[3,5]} & {[0,2]}\end{array}\right]$.

ii). $\left[\begin{array}{cc}{[1,3]} & {[2,3]} \\ {[\varepsilon, \varepsilon]} & {[-3,1]}\end{array}\right] \bar{\oplus}\left[\begin{array}{cc}{[2,2]} & {[-5,-2]} \\ {[1,4]} & {[-1,0]}\end{array}\right]=$

$\left[\begin{array}{ll}{[1,3] \bar{\oplus}[2,2]} & {[2,3] \bar{\oplus}[-5,-2]} \\ {[\varepsilon, \varepsilon] \bar{\oplus}[1,4]} & {[-3,1] \bar{\oplus}[-1,0]}\end{array}\right]=\left[\begin{array}{ll}{[2,3]} & {[2,3]} \\ {[1,4]} & {[-1,1]}\end{array}\right]$.

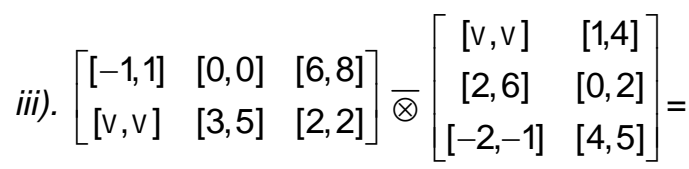

$[[-1,1] \bar{\otimes}[\varepsilon, \varepsilon] \bar{\oplus}[0,0] \bar{\otimes}[2,6] \bar{\oplus}[6,8] \bar{\otimes}[-2,-1]$

$[\varepsilon, \varepsilon] \bar{\otimes}[\varepsilon, \varepsilon] \bar{\oplus}[3,5] \bar{\otimes}[2,6] \bar{\oplus}[2,2] \bar{\otimes}[-2,-1]$

$[-1,1] \bar{\otimes}[1,4] \bar{\oplus}[0,0] \bar{\otimes}[0,2] \bar{\oplus}[6,8] \bar{\otimes}[4,5]]=$

$[\varepsilon, \varepsilon] \bar{\otimes}[1,4] \bar{\oplus}[3,5] \bar{\otimes}[0,2] \bar{\oplus}[2,2] \bar{\otimes}[4,5]$

$[[\varepsilon, \varepsilon] \bar{\oplus}[2,6] \bar{\oplus}[4,7] \quad[0,5] \bar{\oplus}[0,2] \bar{\oplus}[10,13]$

$[\varepsilon, \varepsilon] \bar{\oplus}[5,11] \bar{\oplus}[0,1] \quad[\varepsilon, \varepsilon] \bar{\oplus}[3,7] \bar{\oplus}[6,7]]_{=}$

$\left[\begin{array}{cc}{[4,7]} & {[10,13]} \\ {[5,11]} & {[6,7]}\end{array}\right]$.

Sejalan dengan Teorema 2.1.11 dalam Rudhito (2003) diperoleh diperluasannya untuk versi matriks interval dalam Teorema berikut.

Teorema 1. Pernyataan-pernyataan berikut berlaku untuk sebarang skalar interval $\alpha$ dan $\beta$, dan sebarang matriks interval A, B dan C asalkan operasi yang dimaksud terdefinisi.

i) $\quad(A \bar{\oplus}) \bar{\oplus} C=A \bar{\oplus}(B \bar{\oplus} C)$

ii) $\quad A \bar{\oplus}=B \bar{\oplus} A$

iii) $\quad(A \bar{\otimes} B) \bar{\otimes} C=A \bar{\otimes}(B \bar{\otimes} C)$

iv) $\quad A \bar{\otimes}(B \bar{\oplus} C)=(A \bar{\otimes} B) \bar{\oplus}(A \bar{\otimes} C)$

v) $\quad(A \bar{\oplus} B) \bar{\otimes}=(A \bar{\otimes} C) \bar{\oplus}(B \bar{\otimes} C)$

vi) $\quad \alpha \bar{\otimes} A=A \bar{\otimes} \alpha$

vii) $\quad \alpha \bar{\otimes}(\beta \bar{\otimes} A)=(\alpha \bar{\otimes} \beta) \bar{\otimes} A$

viii) $\quad \alpha \bar{\otimes}(\mathrm{A} \bar{\otimes} \mathrm{B})=(\alpha \bar{\otimes} \mathrm{A}) \bar{\otimes} \mathrm{B}=\mathrm{A} \bar{\otimes}(\alpha \bar{\otimes} \mathrm{B})$

ix) $\quad(\alpha \bar{\oplus} \beta) \bar{\otimes} A=(\alpha \bar{\otimes} A) \bar{\oplus}(\beta \bar{\otimes} A)$

x) $\quad \alpha \bar{\otimes}(A \bar{\oplus} B)=(\alpha \bar{\otimes} A) \bar{\oplus}(\alpha \bar{\otimes} B)$

xi) $\quad A \bar{\oplus} A=A$.

Bukti: Sifat-sifat di atas mengikuti definisi operasi pada Definisi 2 di atas dan sifat-sifat operasi pada $\mathbf{I}(\mathbf{R})_{\max }$.
Didefinisikan matriks $E \in \mathbf{I}(\mathbf{R})_{\max }^{n \times n}$, dengan $(E)_{i j}$ : $=\left\{\begin{array}{l}0, \text { jika } i=j \\ \varepsilon, \text { jika } i \neq j\end{array}\right.$. Didefinisikan pula matriks $\varepsilon \in$ $\mathbf{I}(\mathbf{R}) \underset{\max }{n \times n}$, dengan $(\varepsilon)_{i j}:=\varepsilon$ untuk setiap $i$ dan $j$.

\section{Contoh 2}

Perhatikan bahwa $\mathbf{I}(\mathbf{R})_{\max }^{m \times n}$ tertutup terhadap operasi $\bar{\oplus}$, hal ini akibat dari sifat ketertutupan operasi $\bar{\oplus}$ pada $\mathbf{I}(\mathbf{R})_{\max }$. Selanjutnya dengan memperhatikan sifatsifat pada Teorema 1 di atas nampak bahwa $\left(\mathbf{I}(\mathbf{R})_{\max }^{\operatorname{man}}\right.$, $\bar{\oplus})$ merupakan semigrup komutatif idempotent, sehingga relasi “ $\preceq$ Im” yang didefinisikan pada I(R) ${ }_{\max }^{\operatorname{man}}$ dengan $A \preceq \operatorname{Im} B \Leftrightarrow A \bar{\oplus} B=B$ merupakan urutan parsial. Perhatikan bahwa $A \bar{\oplus} B=B \Leftrightarrow A_{i j} \oplus B_{i j}=$ $\mathrm{B}_{i j} \Leftrightarrow \mathrm{A}_{i j} \preceq_{\mathrm{m} m} \mathrm{~B}_{i j} \Leftrightarrow \mathrm{A}_{i j} \preceq_{\mathrm{m}} \mathrm{B}_{i j}$ dan $\overline{\mathrm{A}_{i j}} \preceq_{\mathrm{m}} \overline{\mathrm{B}_{i j}}$ untuk setiap $i$ dan $j$. Lebih lanjut $\mathbf{I}(\mathbf{R}) \max _{\max } \times \operatorname{merupakan}$ semimodul atas $\mathbf{I}(\mathbf{R})_{\text {max }}$, sedangkan $\left(\mathbf{I}(\mathbf{R})_{\max }^{n \times n}, \bar{\oplus}, \bar{\otimes}\right)$ merupakan semiring idempoten dengan elemen netral adalah matriks $\varepsilon$ dan elemen satuan adalah matriks $\mathrm{E}$. Perhatikan bahwa $\left(\mathbf{I}(\mathbf{R})_{\max }^{n \times n}, \bar{\oplus}, \bar{\otimes}\right)$ bukan semiring komutatif , hal ini sebagai akibat dari $\mathbf{R}_{\text {max }}^{n \times n}$ yang bukan merupakan semiring komutatif.

Karena $\left(\mathbf{I}(\mathbf{R})_{\max }^{m \times n}, \bar{\oplus}\right)$ merupakan semigrup

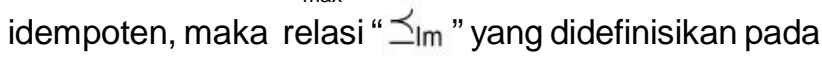
$\mathbf{I}(\mathbf{R})_{\max }^{m \times n}$ konsisten terhadap operasi $\oplus$ konsisten terhadap urutan $\preceq_{\text {Im }}$ dalam $\mathbf{I}(\mathbf{R})_{\max }^{m \times n}$, yaitu jika $A \preceq \operatorname{lm} B$, maka $A \bar{\oplus} \preceq_{I m} B \oplus C, \quad \forall A, B, C \in \mathbf{I}(\mathbf{R})_{\max }^{\operatorname{m} \times n}$. Karena $\left(\mathbf{I}(\mathbf{R})_{\max }^{n \times n}, \bar{\oplus}, \bar{\otimes}\right)$ merupakan semiring idempoten, maka operasi $\bar{\otimes}$ konsisten terhadap urutan $\preceq_{\mathrm{m}}$

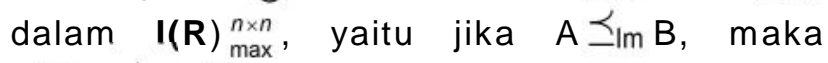
$\mathrm{A} \otimes \mathrm{Q} \preceq_{\mathrm{Im}} \mathrm{B} \otimes \mathrm{Q}, \quad \forall \mathrm{A}, \mathrm{B}, \mathrm{C} \in \mathbf{I}(\mathbf{R})_{\max }^{n \times n}$. Untuk $A \in$ $\mathbf{I}(\mathbf{R})_{\max }^{m \times p} B \in \mathbf{I}(\mathbf{R})_{\max }^{m \times p}$ dan $C \in \mathbf{I}(\mathbf{R})_{\max }^{p \times n}$, berdasarkan sifat distributif, yaitu sifat $v$ ) pada Teorema 1 diatas, berlaku bahwa: jika $A \preceq \operatorname{lm} B$ maka $A \bar{\oplus} B=B \Leftrightarrow(A \bar{\oplus}) \bar{\otimes} C$ $=B \bar{\otimes} C \Leftrightarrow(A \bar{\otimes} C) \bar{\oplus}(B \bar{\otimes} C)=B \bar{\otimes} C \Leftrightarrow A$ $\bar{\otimes} \mathrm{C} \preceq \mathrm{Im} B \bar{\otimes} \mathrm{C}$.

Pangkat $k$ dari matriks $A \in \mathbf{I}(\mathbf{R})_{\max }^{n \times n}$, dalam aljabar max-plus interval didefinisikan dengan: $A^{\otimes 0}=E_{n}$ dan $=$ $\mathrm{A}^{\otimes \kappa}=\mathrm{A} \bar{\otimes} \mathrm{A}^{\otimes \kappa-1}$ untuk $k=1,2, \ldots$

Untuk mempermudah dalam mengoperasikan matriks interval berikut dibahas konsep mengenai interval matriks dari suatu matriks interval.

\section{Definisi 4}

Untuk setiap matriks interval $A \in \mathbf{I}(\mathbf{R})_{\text {max }^{m \times n}}^{\max }$ didefinisikan matriks $\underline{A}=\left(\underline{A_{i j}}\right) \in \mathbf{R}_{\max }^{m \times n}$ dan $\bar{A}=\left(\overline{A_{i j}}\right)$ $\in \mathbf{R}_{\max }^{m \times n}$, yang berturut-turut disebut matriks batas bawah dan matriks batas atas matriks interval $\mathrm{A}$. 


\section{Contoh 3}

Diberikan matriks interval $A=\left[\begin{array}{lll}{[-1,1]} & {[0,0]} & {[6,8]} \\ {[\varepsilon, \varepsilon]} & {[3,5]} & {[2,2]}\end{array}\right]$, maka $\underline{A}=\left[\begin{array}{ccc}-1 & 0 & 6 \\ \varepsilon & 3 & 2\end{array}\right]$ dan $\bar{A}=\left[\begin{array}{lll}1 & 0 & 8 \\ \varepsilon & 5 & 2\end{array}\right]$.

\section{Definisi 5}

Diberikan matriks interval $A \in \mathbf{I}(\mathbf{R})_{\max }^{m \times n}$, dengan $\underline{A}$ dan $\bar{A}$ berturut-turut adalah matriks batas bawah dan matriks batas atasnya. Didefinisikan interval matriks dari $\mathrm{A}$, yaitu $[\underline{\mathrm{A}}, \overline{\mathrm{A}}]=\left\{A \in \mathbf{R}_{\max }^{m \times n} \mid \underline{\mathrm{A}} \preceq_{\mathrm{m}} A\right\}$ dan $\mathbf{I}\left(\mathbf{R}_{\max }^{m \times n}\right)_{\mathrm{b}}=\left\{[\underline{\mathrm{A}}, \overline{\mathrm{A}}] \mid \mathbf{A} \in \mathbf{I}(\mathbf{R})_{\max }^{m \times n}\right\}$.

\section{Contoh 4}

Diberikan matriks interval $A=\left[\begin{array}{lll}{[-1,1]} & {[0,0]} & {[6,8]} \\ {[\varepsilon, \varepsilon]} & {[3,5]} & {[2,2]}\end{array}\right]$. Interval matriks dari $A$ adalah $[\underline{A}, \bar{A}]=$ $\left.\left[\begin{array}{ccc}-1 & 0 & 6 \\ \varepsilon & 3 & 2\end{array}\right],\left[\begin{array}{lll}1 & 0 & 8 \\ \varepsilon & 5 & 2\end{array}\right]\right]$

\section{Definisi 6}

Interval matriks $[\underline{\mathrm{A}}, \overline{\mathrm{A}}]$ dan $[\underline{\mathrm{B}}, \overline{\mathrm{B}}] \in \mathbf{I}\left(\mathbf{R}_{\max }^{m \times n}\right)_{\mathrm{b}}$ dikatakan samajika $\underline{A}=\underline{B}$ dan $\bar{A}=\bar{B}$.

Berdasarkan sifat kekonsistenan relasi urutan $\preceq_{\mathrm{m}}$ dalam matriks, didefinisikan operasi-operasi interval matriks berikut.

i) Diketahui $\alpha \in \mathbf{I}(\mathbf{R})_{\max },[\underline{A}, \overline{\mathrm{A}}],[\underline{B}, \overline{\mathrm{B}}] \in \mathbf{I}\left(\mathbf{R}_{\max }^{m \times n}\right)_{\mathrm{b}}$. Didefinisikan $\alpha \bar{\otimes}[\underline{A}, \bar{A}]=[\underline{\alpha} \otimes \underline{A}, \bar{\alpha} \otimes \bar{A}]$ dan $[\underline{A}, \bar{A}] \bar{\oplus}[\underline{B}, \bar{B}]=[\underline{A} \oplus \underline{B}, \bar{A} \oplus \bar{B}]$

ii) Diketahui $[\underline{A}, \bar{A}] \in \mathbf{I}\left(\mathbf{R}_{\max }^{m \times p}\right)_{b},[\underline{B}, \bar{B}] \in \mathbf{I}\left(\mathbf{R}_{\max }^{p \times n}\right)_{b}$. $\operatorname{Didefinisikan}[\underline{A}, \bar{A}] \bar{\otimes}[\underline{B}, \bar{B}]=[\underline{A} \otimes \underline{B}, \bar{A} \otimes \bar{B}]$.

Teorema 2. Pernyataan-pernyataan berikut berlaku untuk sebarang skalar interval $\alpha$ dan $\beta$, dan sebarang interval matriks $[\underline{A}, \bar{A}],[\underline{B}, \bar{B}]$ dan $[\underline{C}, \bar{C}]$, yang berturut-turut merupakan interval matriks dari matriks interval $\mathrm{A}, \mathrm{B}$ dan $\mathrm{C}$, asalkan operasi yang dimaksud terdefinisi.
i). $\quad([\underline{A}, \bar{A}] \bar{\oplus}[\underline{B}, \bar{B}]) \bar{\oplus}[\underline{C}, \bar{C}]=$
$[\underline{A}, \bar{A}] \oplus([\underline{B}, \bar{B}] \bar{\oplus}[\underline{C}, \bar{C}])$
ii). $[\underline{A}, \bar{A}] \bar{\oplus}[\underline{B}, \bar{B}]=[\underline{B}, \bar{B}] \bar{\oplus}[\underline{A}, \bar{A}]$
iii). $([\underline{A}, \bar{A}] \otimes[\underline{B}, \bar{B}]) \otimes[\underline{C}, \bar{C}]=$
$[\underline{A}, \bar{A}] \bar{\otimes}([\underline{B}, \bar{B}] \bar{\otimes}[\underline{C}, \bar{C}])$
iv). $[\underline{A}, \bar{A}] \bar{\otimes}([\underline{B}, \bar{B}] \oplus[\underline{C}, \bar{C}])=$
$([\underline{A}, \bar{A}] \bar{\otimes}[\underline{B}, \bar{B}]) \bar{\oplus}([\underline{A}, \bar{A}] \bar{\otimes}[\underline{C}, \bar{C}])$
v). $([\underline{A}, \bar{A}] \oplus[\underline{B}, \bar{B}]) \bar{\otimes}[\underline{C}, \bar{C}]=([\underline{A}, \bar{A}]$
$\bar{\otimes}[\underline{\mathrm{C}}, \overline{\mathrm{C}}]) \bar{\oplus}([\underline{\mathrm{B}}, \overline{\mathrm{B}}] \bar{\otimes}[\underline{\mathrm{C}}, \overline{\mathrm{C}}])$
vi). $\alpha \bar{\otimes}[\underline{A}, \bar{A}]=[\underline{A}, \bar{A}] \bar{\otimes} \alpha$
vii). $\alpha \bar{\otimes}(\beta \bar{\otimes}[\underline{A}, \bar{A}])=(\alpha \bar{\otimes} \beta) \bar{\otimes}[\underline{A}, \bar{A}]$

viii). $\alpha \bar{\otimes}([\underline{\mathrm{A}}, \overline{\mathrm{A}}] \bar{\otimes}[\underline{\mathrm{B}}, \overline{\mathrm{B}}])=(\alpha \bar{\otimes}[\underline{\mathrm{A}}, \overline{\mathrm{A}}]) \bar{\otimes}[\underline{\mathrm{B}}, \overline{\mathrm{B}}]$ $=[\underline{A}, \bar{A}] \bar{\otimes}(\alpha \bar{\otimes}[\underline{B}, \bar{B}])$

ix). $(\alpha \bar{\oplus} \mathrm{b}) \bar{\otimes}[\underline{\mathrm{A}}, \overline{\mathrm{A}}]=(\mathrm{a} \bar{\otimes}[\underline{\mathrm{A}}, \overline{\mathrm{A}}]) \bar{\oplus}(\beta \bar{\otimes}[\underline{\mathrm{A}}, \overline{\mathrm{A}}])$

$\mathrm{x}) . \quad \alpha \bar{\otimes}([\underline{\mathrm{A}}, \overline{\mathrm{A}}] \bar{\oplus}[\underline{\mathrm{B}}, \overline{\mathrm{B}}])=(\alpha \bar{\otimes}[\underline{\mathrm{A}}, \overline{\mathrm{A}}]) \bar{\oplus}$ $(\alpha \bar{\otimes}[\mathrm{B}, \overline{\mathrm{B}}])$

xi). $[\underline{A}, \bar{A}] \bar{\oplus}[\underline{A}, \bar{A}]=[\underline{A}, \bar{A}]$.

Bukti: Sifat-sifat di atas mengikuti definisi operasi pada interval matriks di atas dan sifat-sifat operasi pada matriks atas aljabar max-plus $\mathbf{R}_{\max }$.

Untuk setiap $[\underline{\mathrm{A}}, \overline{\mathrm{A}}],[\underline{\mathrm{B}}, \overline{\mathrm{B}}] \in \mathbf{I}\left(\mathbf{R}_{\max }^{m \times n}\right)_{\mathrm{b}}$ dan $\mathrm{a} \alpha \in$ $\mathbf{I}(\mathbf{R})_{\max }$ berlaku $\underline{A} \preceq_{m} \bar{A}$, dan $\underline{\alpha} \preceq_{m} \bar{\alpha}$. Karena operasi $\oplus$ dan operasi perkalian skalar $\otimes$ pada semimodul $\mathbf{R}_{\max }^{m \times n}$ atas $\mathbf{R}_{\max }$ konsisten terhadap urutan “ $\preceq_{\mathrm{m}}$ ”, maka berlaku $\underline{A} \oplus \underline{B} \preceq_{m} \bar{A} \oplus \bar{B}$ dan $\underline{\alpha} \otimes \underline{A} \preceq_{m} \bar{\alpha} \otimes \bar{A}$. Jadi $[\underline{A} \AA \underline{\mathrm{A}}, \overline{\mathrm{A}} \oplus \overline{\mathrm{B}}]$ dan $[\underline{\alpha} \otimes \underline{\mathrm{A}}, \bar{\alpha} \otimes \overline{\mathrm{A}}]$ merupakan interval-interval matriks. Jadi $\mathbf{I}\left(\mathbf{R}_{\max }^{\mathrm{m} \times \mathrm{n}}\right)_{\mathrm{b}}$ tertutup terhadap operasi $\bar{\oplus}$ dan perkalian skalar $\bar{\otimes}$ seperti yang didefinisikan di atas. Selanjutnya sesuai dengan definisi operasi pada interval matriks di atas dan sifat-sifat pada Teorema 2 nampak bahwa $\mathbf{I}\left(\mathbf{R}_{\max }^{\mathrm{m} \times n}\right)_{\mathrm{b}}$ merupakan semimodul atas $\mathbf{I}(\mathbf{R})_{\max }$.

Untuk setiap $[\underline{A}, \bar{A}],[\underline{B}, \bar{B}] \in \mathbf{I}\left(\mathbf{R}_{\max }^{\mathrm{n} \times n}\right)_{b}$ berlaku $\underline{A} \preceq_{m} \bar{A}$ dan $\underline{B} \preceq_{m} \bar{B}$. Karena operasi perkalian $\otimes$ pada semiring konsisten terhadap urutan " $\preceq_{\mathrm{m}}$ ", maka $\underline{A} \otimes \underline{B} \otimes$. Jadi $[\underline{A} \oplus \underline{B}, \bar{A} \oplus \bar{B}]$ merupakan interval matriks. Jadi $\mathbf{I}\left(\mathbf{R}_{\max }^{\mathrm{n} \times n}\right)_{\mathrm{b}}$ tertutup terhadap operasi perkalian seperti yang didefinisikan di atas. Selanjutnya menurut sifat-sifat pada Teorema 2 nampak bahwa bahwa $\left(\mathbf{I}\left(\mathbf{R}_{\max }^{\mathrm{n} \times \mathrm{n}}\right)_{\mathrm{b}}, \bar{\oplus}, \bar{\otimes}\right)$ merupakan semiring idempoten dengan elemen netral adalah interval matriks $[\varepsilon, \varepsilon]$ dan elemen satuan adalah interval matriks $[E, E]$.

Teorema 3. Untuk setiap $\mathrm{A}$ dan $\mathrm{B} \in \mathbf{I}(\mathbf{R})_{\max }^{m \times n}$, berlaku

i). $\underline{\alpha \bar{\otimes} \mathrm{A}}=\underline{\alpha} \otimes \underline{\mathrm{A}}$ dan $\overline{\alpha \bar{\otimes} \mathrm{A}}=\bar{\alpha} \otimes \overline{\mathrm{A}}, i i) \underline{\mathrm{A} \oplus \bar{\oplus} \mathrm{B}}=$ $\underline{A} \oplus \underline{B}$ dan $\overline{A \bar{\oplus} B}=\bar{A} \oplus \bar{B}$

\section{Bukti:}

i). Karena $(\alpha \bar{\otimes} A)_{i j}=[\underline{\alpha}, \bar{\alpha}] \bar{\otimes}\left[A_{i j}, \overline{A_{i j}}\right]=$ $\left[\underline{\alpha} \otimes \mathrm{A}_{i j}, \bar{\alpha} \otimes \overline{\mathrm{A}_{i j}}\right]$, maka $\underline{\alpha \bar{\otimes}} \mathrm{A}_{i j}=\underline{\alpha \otimes} \mathrm{A}_{i j}$ dan $\overline{\alpha \bar{\otimes} \mathrm{A}_{i j}}=\bar{\alpha} \otimes \overline{\mathrm{A}_{i j}}$, untuk setiap $i$ dan $j$, sehingga $=\underline{\alpha \bar{\otimes} \mathrm{A}}=\underline{\alpha} \otimes \underline{\mathrm{A}}$ dan $\overline{\alpha \bar{\otimes} \mathrm{A}}=\bar{\alpha} \otimes \overline{\mathrm{A}}$.

ii). Karena Karena $(A \oplus B)_{i j}=A_{i j} \oplus B_{i j}=$

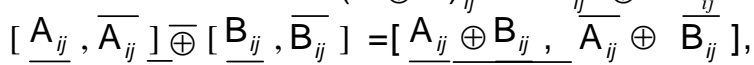

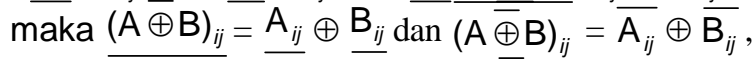
untuk setiap $i$ dan $\bar{j}$, sehingga $\bar{A} \oplus \mathrm{B}=\underline{A} \oplus \underline{B}$ dan $\overline{A \bar{\oplus} B}=\bar{A} \oplus \bar{B}$.

Teorema 4. Untuk setiap $A$ dan $B \in \mathbf{I}(\mathbf{R})_{\max }^{n \times n}$, berlaku $\underline{A \bar{\otimes} B}=\underline{A} \otimes \underline{B}$ dan $\overline{A \bar{\otimes} B}=\bar{A} \otimes \bar{B}$. 
Bukti:

Karena $\quad(\mathrm{A} \bar{\otimes} \mathrm{B})_{i j}=\quad \bigoplus_{k=1}^{n} \mathrm{~A}_{i j} \bar{\otimes} \mathrm{B}_{i j}=\frac{n}{\bigoplus_{k=1}^{n}}$ $\left[\underline{\mathrm{A}_{i k}}, \overline{\mathrm{A}_{i k}}\right] \bar{\otimes}\left[\underline{\mathrm{B}_{k j}}, \overline{\mathrm{B}_{k j}}\right]=\overline{\bigoplus_{k=1}^{n}}\left[\underline{\mathrm{A}_{i k}} \ddot{\ddot{\mathrm{B}}} \underline{\mathrm{B}_{k j}}, \overline{\mathrm{A}_{i k}} \ddot{\mathrm{A}} \overline{\mathrm{B}_{k j}}\right]=$ $\left[\bigoplus_{k=1}^{n} \underline{\mathrm{A}_{i k}} \otimes \underline{\mathrm{B}_{k j}}, \quad \bigoplus_{k=1}^{n} \overline{\mathrm{A}_{i k}} \otimes \overline{\mathrm{B}_{k j}}\right], \quad$ maka $\quad \underline{(\mathrm{A} \bar{\otimes} \mathrm{B})_{i j}}=$ $\underline{\bigoplus_{k=1}^{n}} \underline{\mathrm{A}_{i k}} \otimes \underline{\mathrm{B}_{k j}}$ dan $\overline{(\mathrm{A} \bar{\otimes} \mathrm{B})_{i j}}=\bigoplus_{k=1}^{n} \overline{\mathrm{A}_{i k}} \ddot{\mathrm{A}} \overline{\mathrm{B}_{k j}}$, untuk setiap $i$ dan $j$, sehingga $\underline{A \bar{\otimes} B}=\underline{A} \otimes \underline{B}$ dan $\overline{A \bar{\otimes} B}=$ $\overline{\mathrm{A}} \otimes \overline{\mathrm{B}}$.

Teorema 5. Semimodul $\mathbf{I}(\mathbf{R})_{\max }^{m \times n}$ atas $\mathbf{I}(\mathbf{R})_{\max }$ isomorfis dengan semimodul $\mathbf{I}\left(\mathbf{R}_{\max }^{m \times n}\right)_{b}$ atas $\mathbf{I}(\mathbf{R})_{\max }$

\section{Bukti:}

Didefinisikan pemetaan $f: \mathbf{I}(\mathbf{R})_{\max }^{m \times n} \rightarrow \mathbf{I}\left(\mathbf{R}_{\max }^{m \times n}\right)^{\mathrm{b}}, f(\mathrm{~A})=$ $[\underline{\mathrm{A}}, \overline{\mathrm{A}}], \forall \mathrm{A} \in \mathbf{I}(\mathbf{R})_{\max }^{m \times n}$.

i) Ambil sembarang $A$ dan $B \in \mathbf{I}(\mathbf{R})_{\max }^{m \times n}$, sedemikian hingga $A=B$. Karena $A=B$, maka $\underline{A_{i j}}=\underline{B_{i j}}$ dan $\overline{A_{i j}}$ $=\overline{B_{i j}}$ untuk setiap $i$ dan $j$. Hal ini berarti $\underline{A}=\underline{B}$ dan $\overline{\mathrm{A}}=\overline{\mathrm{B}}$, sehingga $[\underline{\mathrm{A}}, \overline{\mathrm{A}}]=[\underline{\mathrm{B}}, \overline{\mathrm{B}}]$. Jadi $f(\mathrm{~A})=f(\mathrm{~B})$ yang berarti $f$ merupakan pemetaan.

ii) Ambil sembarang $[\underline{A}, \bar{A}] \in \mathbf{I}\left(\mathbf{R}_{\max }^{m \times n}\right)^{\mathrm{b}}$, maka $\underline{\mathrm{A}}, \overline{\mathrm{A}} \in \mathbf{R}_{\max }^{m \times n}$, sehingga $\left[\underline{\mathrm{A}}_{i j}, \overline{\mathrm{A}}_{i j}\right] \in \mathbf{I}(\mathbf{R})_{\max }, \forall i$ dan j. Jadi terdapat $A \in \mathbf{I}(\mathbf{R})_{\max }^{m \times n}$, dengan $A_{i j}=\left[\underline{A}_{i j}, \bar{A}_{i j}\right]$, $\forall i$ dan $j$. Jadi untuk setiap $[\underline{\mathrm{A}}, \overline{\mathrm{A}}] \in \mathbf{I}\left(\mathbf{R}_{\max }^{m \times n}\right)^{\mathrm{b}}$, terdapat $\mathrm{A} \in \mathbf{I}(\mathbf{R})_{\max }^{m \times n}$ sedemikian hingga $f(\mathrm{~A})=$ $[\underline{A}, \overline{\mathrm{A}}]$, yang berarti $f$ surjektif.

iii) Ambil sembarang $A$ dan $B \in \mathbf{I}(\mathbf{R})_{\max }^{m \times n}$, sedemikian hingga $f(A)=f(B)$, yaitu $[\underline{A}, \bar{A}]=[\underline{B}, \bar{B}]$. Karena $[\underline{\mathrm{A}}, \overline{\mathrm{A}}]=[\underline{\mathrm{B}}, \overline{\mathrm{B}}]$, maka $\underline{\mathrm{A}}=\underline{\mathrm{B}}$ dan $\overline{\mathrm{A}}=\overline{\mathrm{B}} \cdot$ Hal ini berarti untuk setiap $i$ dan $j$ berlaku $\underline{A}_{i j}=\underline{\mathrm{B}}_{i j} \operatorname{dan} \overline{\mathrm{A}}_{i j}$ $=\overline{\mathrm{B}}_{i j}$, sehingga $\left[\underline{\mathrm{A}}_{i j}, \overline{\mathrm{A}}_{i j}\right]=\left[\underline{\mathrm{B}}_{i j}, \overline{\mathrm{B}}_{i j}\right]$. Jadi $\mathrm{A}=\mathrm{B}$, yang berarti $f$ injektif.

iv) Ambil sembarang $A$ dan $B \in \mathbf{I}(\mathbf{R})_{\max }^{m \times n}$ dan sembarang $\alpha \in \mathbf{I}(\mathbf{R})_{\max }$, maka $f(\alpha \bar{\otimes} \mathrm{A})=[\underline{\alpha \bar{\otimes} \mathrm{A}}, \overline{\alpha \bar{\otimes} \mathrm{A}}]=$ $[\underline{\alpha} \otimes \underline{\mathrm{A}}, \bar{\alpha} \otimes \overline{\mathrm{A}}]=[\underline{\alpha}, \bar{\alpha}] \bar{\otimes}[\underline{\mathrm{A}}, \overline{\mathrm{A}}]=\alpha \bar{\otimes} f(\mathrm{~A})$ dan $f(\mathrm{~A} \oplus \mathrm{B})=[\underline{(\mathrm{A} \bar{\oplus} \mathrm{B}}), \overline{\mathrm{A} \oplus \mathrm{B}}]=[\underline{\mathrm{A}} \oplus \underline{\mathrm{B}}, \overline{\mathrm{A}} \oplus \overline{\mathrm{B}}]$ $=[\underline{\mathrm{A}}, \overline{\mathrm{A}}] \bar{\oplus}[\underline{\mathrm{B}}, \overline{\mathrm{B}}]=f(\mathrm{~A}) f(\mathrm{~B})$.
Karena semimodul $\mathbf{I}(\mathbf{R})_{\max }^{m \times n}$ isomorfis dengan semimodul $\mathbf{I}\left(\mathbf{R}_{\max }^{m \times n}\right)_{\mathrm{b}}$, maka dapat disimpulkan bahwa untuk setiap matriks interval $A \in \mathbf{I}(\mathbf{R})_{\max }^{m \times n}$ selalu dapat ditentukan dengan tunggal interval matriks $[\underline{A}, \bar{A}] \in$ $\mathbf{I}\left(\mathbf{R}_{\max }^{m \times n}\right)_{b}$, demikian juga sebaliknya. Dengan demikiaan matriks interval $A \in \mathbf{I}(\mathbf{R})$ dapat dipandang sebagai interval matriks $[\underline{A}, \bar{A}] \in \mathbf{I}\left(\mathbf{R}_{\max }^{m \times n}\right)_{b}$. Interval matriks $[\underline{\mathrm{A}}, \overline{\mathrm{A}}] \in \mathbf{I}\left(\mathbf{R}_{\max }^{m \times n}\right)_{\mathrm{b}}$ disebut interval matriks yang bersesuaian dengan matriks interval $\mathbf{A} \in \mathbf{I}(\mathbf{R})_{\max }^{n \times n}$ dan dilambangkan dengan $A \approx[\underline{A}, \bar{A}]$. Dapat disimpulkan juga bahwa $\alpha \bar{\otimes} \mathrm{A} \approx[\underline{\alpha} \otimes \underline{A}, \bar{\alpha} \otimes \bar{A}]$ dan $A \bar{\oplus} B \approx$ $[\underline{\mathrm{A}} \oplus \underline{\mathrm{B}}, \overline{\mathrm{A}} \oplus \overline{\mathrm{B}}]$.

Teorema 6. Semiring $\left(\mathbf{I}(\mathbf{R})_{\max }^{n \times n}, \bar{\oplus}, \bar{\otimes}\right)$ isomorfis dengan semiring $\left(\mathbf{I}\left(\mathbf{R}_{\max }^{n \times n}\right)_{b}, \bar{\oplus}, \bar{\otimes}\right)$.

Bukti: Didefinisikan pemetaan $f: \mathbf{I}(\mathbf{R})_{\text {max }}^{n \times n} \rightarrow$ $\mathbf{I}\left(\mathbf{R}_{\max }^{n \times n}\right)_{\mathrm{b}}$ dengan $f\left((\mathrm{~A})=[\underline{\mathrm{A}}, \overline{\mathrm{A}}], \forall \mathbf{A} \in \mathbf{I}(\mathbf{R})_{\max }^{n \times n}\right.$. Menurut pembuktian Teorema 5 di atas pemetaan $f$ merupakan pemetaan bijektif. Ambil sembarang Adan $B \in \mathbf{I}(\mathbf{R})_{\max }^{n \times n}$, maka seperti pada pembuktian pada Teorema 5 di atas diperoleh $f(\mathrm{~A} \oplus \mathrm{B})=f(\mathrm{~A}) \bar{\oplus} f(\mathrm{~B})$. Selanjutnya $f(A \bar{\otimes} B)=[(\underline{A \otimes} \bar{\otimes}), \overline{A \bar{\otimes} B}=[\underline{A} \otimes \underline{B}$, $\overline{\mathrm{A}} \otimes \overline{\mathrm{B}}]=[\underline{\mathrm{A}}, \overline{\mathrm{A}}] \bar{\otimes}[\underline{\mathrm{B}}, \overline{\mathrm{B}}]=f(\mathrm{~A}) \bar{\otimes} f(\mathrm{~B})$. Jadi terbukti $f$ merupakan suatu isomorfisma semiring. Dengan kata lain semiring $\mathbf{I}(\mathbf{R})_{\max }^{n \times n}$ isomorfis dengan semiring $\mathbf{I}\left(\mathbf{R}_{\max }^{n \times n}\right)$.

Karena semiring $\mathbf{I}(\mathbf{R})_{\max }^{n \times n}$ isomorfis dengan semiring $\mathbf{I}\left(\mathbf{R}_{\max }^{n \times n}\right)_{b}$, maka dapat disimpulkan bahwa $A \bar{\otimes} B \approx[\underline{A} \otimes \underline{B}, \bar{A} \otimes \bar{B}]$. Untuk perkalian matriks interval $\mathrm{A} \in \mathbf{I}(\mathbf{R})_{\max }^{m \times p}$ dan $\mathrm{B} \in \mathbf{I}(\mathbf{R})_{\max }^{p \times n}$ juga berlaku $\mathrm{A} \otimes \mathrm{B}$ $\approx[\underline{A} \otimes \underline{B}, \bar{A} \otimes \bar{B}] . ~ H a l$ ini dapat dijelaskan sebagai berikut. Matriks interval $A \in \mathbf{I}(\mathbf{R})_{\max }^{\operatorname{map}}$ dan $\mathrm{B} \in \mathbf{I}(\mathbf{R})$ dapat diperbesar ukurannya dengan menambahkan sejumlah unsur e sedemikian hingga membentuk matriks interval $\mathrm{A}^{\#}$ dan $\mathrm{B}^{\#} \in \mathbf{I}(\mathbf{R})_{\max }^{k \times k}$, dengan $k=\max (m, p, n)$. Matriks A dan $B$ berturut-turut merupakan submatriks $A^{\#}$ dan $B^{\#}$ yang letaknya di sebelah kiri atas, yaitu

$A^{\#}=\left[\begin{array}{ll}A & \varepsilon \\ \varepsilon & \varepsilon\end{array}\right], B^{\#}=\left[\begin{array}{ll}B & \varepsilon \\ \varepsilon & \varepsilon\end{array}\right]$, sehingga $A^{\#} \bar{\otimes} B^{\#}=$ $\left[\begin{array}{cc}\mathrm{A} \bar{\otimes} \mathrm{B} & \boldsymbol{\varepsilon} \\ \boldsymbol{\varepsilon} & \boldsymbol{\varepsilon}\end{array}\right] \in \mathbf{I}(\mathbf{R})_{\max }^{k \times k}$, di mana $\mathrm{A} \bar{\otimes} \mathrm{B} \in \mathbf{I}(\mathbf{R})_{\max }^{m \times n}$. Karena semiring $\mathbf{I}(\mathbf{R})_{\max }^{k \times k}$ isomorfis dengan semiring $\mathbf{I}\left(\mathbf{R}_{\max }^{k \times k}\right)_{b}$, maka $A^{\#} \bar{\otimes} B^{\#} \approx\left[\underline{A^{\#} \otimes B^{\#}}, \overline{A^{\#}} \otimes \overline{B^{\#}}\right]$, yang berakibat bahwa $\mathrm{A} \bar{\otimes} \mathrm{B} \approx[\underline{\mathrm{A}} \otimes \underline{\mathrm{B}}, \overline{\mathrm{A}} \otimes \overline{\mathrm{B}}] \in \mathbf{I}\left(\mathbf{R}_{\max }^{m \times n}\right)_{\mathrm{b}}$. 


\section{Contoh 3}

$A=\left[\begin{array}{ccc}{[-1,1]} & {[0,0]} & {[6,8]} \\ {[\varepsilon, \varepsilon]} & {[3,5]} & {[2,2]}\end{array}\right], \quad \underline{A}=\left[\begin{array}{ccc}-1 & 0 & 6 \\ \varepsilon & 3 & 2\end{array}\right], \quad \bar{A}=$ $\left[\begin{array}{lll}1 & 0 & 8 \\ \varepsilon & 5 & 2\end{array}\right] . \quad B=\left[\begin{array}{cc}{[\varepsilon, \varepsilon]} & {[1,4]} \\ {[2,6]} & {[0,2]} \\ {[-2,-1]} & {[4,5]}\end{array}\right], \underline{B}=\left[\begin{array}{cc}\varepsilon & 1 \\ 2 & 0 \\ -2 & 4\end{array}\right], \bar{B}=$ $\left[\begin{array}{cc}\varepsilon & 4 \\ 6 & 2 \\ -1 & 5\end{array}\right], \underline{A} \otimes \underline{B}=\left[\begin{array}{cc}4 & 10 \\ 5 & 6\end{array}\right], \quad \bar{A} \otimes \bar{B}=\left[\begin{array}{cc}7 & 13 \\ 11 & 7\end{array}\right]$. Perhatikan bahwa $A \bar{\otimes} B \approx[\underline{A} \otimes \underline{B}, \bar{A} \otimes \bar{B}]=$ $\left[\left[\begin{array}{cc}4 & 10 \\ 5 & 6\end{array}\right],\left[\begin{array}{cc}7 & 13 \\ 11 & 7\end{array}\right]\right]$, sehingga $A \bar{\otimes} \quad B=$ $\left[\begin{array}{cc}{[4,7]} & {[10,13]} \\ {[5,11]} & {[6,7]}\end{array}\right]$.

\section{KESIMPULAN}

Dari pembahasan di atas dapat disimpulkan bahwa himpunan semua matriks interval yang dilengkapi dengan operasi perkalian skalar max-plus dan penjumlahan max-plus merupakan semimodul. Himpunan semua matriks persegi atas aljabar maxplus interval yang dilengkapi dengan operasi penjumlahan max-plus dan perkalian max-plus merupakan semiring idempoten. Semimodul himpunan semua matriks interval isomorfis dengan semimodul himpunan interval matriks yang bersesuaian. Semiring himpunan semua matriks interval persegi isomorfis dengan semiring himpunan interval matriks persegi yang bersesuaian. Sebagai akibatnya, operasi-operasi pada matriks interval dapat dilakukan melalui interval matriksnya.

\section{UCAPAN TERIMAKASIH}

Penulis mengucapkan terima kasih kepada Yayasan Sanata Dharma Yogyakarta yang telah membiayai studi dan penelitian ini di Program S3 Matematika FMIPA Universitas Gadjah Mada, Yogyakarta dengan Nomor Kontrak K-1260/Y-15/3-15/ IX/2007.

\section{DAFTAR PUSTAKA}

Bacelli, F et al. 2001. Synchronization and Linearity. New York: John Wiley dan Sons.

Chanas, S. \& Zielinski, P. 2001. Critical path analysis in the network with fuzzy activity times. Fuzzy Sets and Systems. 122: 195-204.

Krivulin, N.K. 2001. Evaluation of Bounds on Service Cycle Times in Acyclic Fork-Join Queueing Networks. International Journal of Computing Anticipatory Systems 9: 94-109.

Litvinov, G.L. \& Sobolevskii, A.N. 2001. Idempotent Interval Anaysis and Optimization Problems. Reliab. Comput 7: 353377.

Lüthi, J. \& Haring, G. 1997. Fuzzy Queueing Network Models of Computing Systems. Proceedings of the 13th UK Performance Engineering Workshop, Ilkley, UK, Edinburgh University Press, July 1997.

Rudhito, A. 2003. Sistem Linear Max-Plus Waktu-Invariant. Tesis: Program Pascasarjana, Yogyakarta: UGM.

Rudhito, A. Wahyuni, S. Suparwanto, A. \& Susilo, F. 2008. Aljabar Max-Plus Interval. Prosiding Seminar Nasional Matematika S3 UGM. Yogyakarta. 31 Mei 2008.

Susilo, F. 2006. Himpunan dan Logika Kabur serta Aplikasinya. Edisi kedua. Yogyakarta: Graha IImu. 